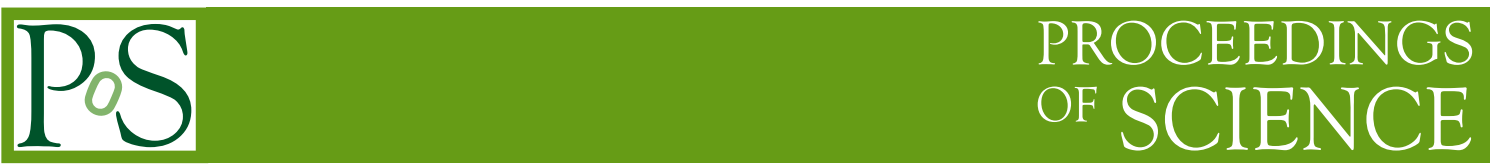

\title{
Electroweak corrections to hadronic event shapes
}

\author{
Ansgar Denner ${ }^{a}$, Stefan Dittmaier ${ }^{b *}$, Thomas Gehrmann ${ }^{c}$ and Christian Kurz ${ }^{a, c \dagger}$ \\ ${ }^{a}$ Paul Scherrer Institut, Würenlingen und Villigen, CH-5232 Villigen PSI, Switzerland \\ ${ }^{b}$ Albert-Ludwigs-Universität Freiburg, Physikalisches Institut, D-79104 Freiburg, Germany \\ ${ }^{c}$ Institut für Theoretische Physik, Universität Zürich, CH-8057 Zürich, Switzerland \\ E-mail: ansgar.dennerapsi.ch, \\ stefan.dittmaier@physik.uni-freiburg.de, \\ thomas.gehrmann@physik.unizh.ch
}

\begin{abstract}
We report on a recent calculation of the electroweak $\mathscr{O}\left(\alpha^{3} \alpha_{\mathrm{s}}\right)$ corrections to 3-jet production and related event-shape observables at $\mathrm{e}^{+} \mathrm{e}^{-}$colliders. The calculation properly accounts for the experimental photon isolation criteria and for the corrections to the total hadronic cross section. Corrections to the normalised event-shape distributions, which are exemplarily discussed here for the thrust distribution at LEP and linear-collider energies, turn out to be at the few-per-cent level and show remnants of the radiative return to the $\mathrm{Z}$ pole even after inclusion of appropriate cuts.
\end{abstract}

RADCOR 2009 - 9th International Symposium on Radiative Corrections (Applications of Quantum Field Theory to Phenomenology)

October 25-30 2009

Ascona, Switzerland

\footnotetext{
*Speaker.

$\dagger$ This work was supported in part by the Swiss National Science Foundation (SNF) under contracts 200020-116756, 200020-124773 and 200020-126691 and by the European Community's Marie-Curie Research Training Network HEPTOOLS under contract MRTN-CT-2006-035505.
} 


\section{Introduction}

Precision QCD studies at $\mathrm{e}^{+} \mathrm{e}^{-}$colliders rely on the measurement of the 3-jet production cross section and related event-shape observables. The deviation from simple 2-jet configurations is proportional to the strong coupling constant $\alpha_{\mathrm{s}}$, so that by comparing the measured 3-jet rate and event shapes (see, e.g., Ref. [1]) with the theoretical predictions, one can determine $\alpha_{\mathrm{s}}$. Including electroweak coupling factors, the leading-order (LO) contribution to this process is of order $\alpha^{2} \alpha_{\mathrm{s}}$.

Owing to recent calculational progress, the QCD predictions for event shapes $[2,3]$ and 3jet production $[4,5]$ are now accurate to next-to-next-to-leading order (NNLO, $\alpha^{2} \alpha_{\mathrm{s}}^{3}$ ) in QCD perturbation theory. Inclusion of these corrections results in an estimated residual uncertainty of the QCD prediction from missing higher orders at the level of well below 5\% for the event-shape distributions, and around $1 \%$ for the 3 -jet cross section. Using these results (combined [6] with the previously available resummed expressions), new determinations of $\alpha_{s}$ from event-shape and jetproduction data were performed, resulting in a considerable improvement of the theory uncertainty to $3 \%$ from event shapes [7] and below $2 \%$ from jet rates [8]. A further improvement can be anticipated for the event shapes from the resummation of subleading logarithmic corrections [9].

At this level of theoretical precision, higher-order electroweak effects could be of comparable magnitude. Until recently, only partial calculations of electroweak corrections to 3-jet production and event shapes were available [10], which can not be compared with experimental data directly. In Ref. [11] we have presented the first calculation of the next-to-leading order (NLO) electroweak $\left(\alpha^{3} \alpha_{s}\right)$ corrections to 3 -jet observables in $\mathrm{e}^{+} \mathrm{e}^{-}$collisions including the quark-antiquark-photon $(q \bar{q} \gamma)$ final states. Note that the QCD corrections to these final states are of the same perturbative order as the genuine electroweak corrections to quark-antiquark-gluon $(q \bar{q} \mathrm{~g})$ final states. Since photons produced in association with hadrons can never be fully isolated, both types of corrections have to be taken into account. In this short article we supplement the results of Ref. [11], where the differential thrust distribution and the 3-jet rate are discussed at the Z-boson resonance, by showing results on the thrust distribution for higher LEP and linear-collider energies. An extensive discussion of event-shape observables and more details of our calculation will be published elsewhere.

\section{Corrections to jet observables}

Event-shape measurements at LEP usually rely on a standard set of six variables $y$, defined for example in Ref. [12]: thrust $T, C$-parameter, heavy jet mass $\rho$, wide and total jet broadenings $B_{\mathrm{W}}$ and $B_{\mathrm{T}}$, and 2-to-3-jet transition parameter in the Durham algorithm $Y_{3}$. The experimentally measured event-shape distribution $(\mathrm{d} \sigma / \mathrm{d} y) / \sigma_{\text {had }}$ is normalised to the total hadronic cross section $\sigma_{\text {had. }}$. In the perturbative expansion, it turns out to be most appropriate to consider the expansion of this ratio, which reads to NNLO in QCD and NLO in the electroweak theory

$$
\frac{1}{\sigma_{\mathrm{had}}} \frac{\mathrm{d} \sigma}{\mathrm{d} y}=\left(\frac{\alpha_{\mathrm{s}}}{2 \pi}\right) \frac{\mathrm{d} \bar{A}}{\mathrm{~d} y}+\left(\frac{\alpha_{\mathrm{s}}}{2 \pi}\right)^{2} \frac{\mathrm{d} \bar{B}}{\mathrm{~d} y}+\left(\frac{\alpha_{\mathrm{s}}}{2 \pi}\right)^{3} \frac{\mathrm{d} \bar{C}}{\mathrm{~d} y}+\left(\frac{\alpha}{2 \pi}\right) \frac{\mathrm{d} \delta_{\gamma}}{\mathrm{d} y}+\left(\frac{\alpha_{s}}{2 \pi}\right)\left(\frac{\alpha}{2 \pi}\right) \frac{\mathrm{d} \delta_{\mathrm{EW}}}{\mathrm{d} y}
$$

where the fact is used that the perturbative expansion of $\sigma_{\text {had }}$ starts at order $\alpha^{2}$. The calculation of the QCD coefficients $\bar{A}, \bar{B}$, and $\bar{C}$ is described in Refs. [2, 3]. The LO purely electromagnetic 
contribution $\delta_{\gamma}$ arises from tree-level $q \bar{q} \gamma$ final states without a gluon. The NLO electroweak coefficient $\delta_{\mathrm{EW}}$ receives contributions from the $\mathscr{O}(\alpha)$ correction to the hadronic cross section and from the genuine $\mathscr{O}\left(\alpha^{3} \alpha_{s}\right)$ contribution to the event-shape distribution,

$$
\sigma_{\text {had }}=\sigma_{0}\left[1+\left(\frac{\alpha}{2 \pi}\right) \delta_{\sigma, 1}\right], \quad \frac{1}{\sigma_{0}} \frac{\mathrm{d} \sigma}{\mathrm{d} y}=\left(\frac{\alpha_{\mathrm{s}}}{2 \pi}\right) \frac{\mathrm{d} \bar{A}}{\mathrm{~d} y}+\left(\frac{\alpha_{\mathrm{s}}}{2 \pi}\right)\left(\frac{\alpha}{2 \pi}\right) \frac{\mathrm{d} \delta_{\bar{A}}}{\mathrm{~d} y},
$$

such that

$$
\frac{\mathrm{d} \delta_{\mathrm{EW}}}{\mathrm{d} y}=\frac{\mathrm{d} \delta_{\bar{A}}}{\mathrm{~d} y}-\delta_{\sigma, 1} \frac{\mathrm{d} \bar{A}}{\mathrm{~d} y}
$$

yields the full NLO electroweak correction. Both terms are to be evaluated with the same eventselection cuts. As shown in the following, many of the numerically dominant contributions, especially from initial-state radiation, cancel in this difference.

In the experimental measurement of 3-jet observables at $\mathrm{e}^{+} \mathrm{e}^{-}$centre-of-mass (CM) energy $\sqrt{s}$, several cuts are applied to reduce the contributions from photonic radiation. In our calculation, we apply the criteria used in the ALEPH analysis [12]. Very similar criteria were also applied by the other LEP experiments. Particles (including $b$ quarks) contribute to the final state only if they are within the detector acceptance, defined by the production angle relative to the beam direction, $|\cos \theta|<0.965$. Events are accepted if the reconstructed invariant mass squared $s^{\prime}$ of the final-state particles is larger than $s_{\mathrm{cut}}=0.81 \mathrm{~s}$. To reduce the contribution from hard photon radiation, the final-state particles are clustered into jets using the Durham algorithm with resolution parameter $y_{\text {cut }}=0.002$. If one of the resulting jets contains a photon carrying a fraction $z_{\gamma}>z_{\gamma \text {,cut }}=0.9$ of the jet energy, it is considered to be an isolated photon, and the event is discarded. The event-shape variables are then computed in the CM frame of the final-state momenta, which can be boosted relative to the $\mathrm{e}^{+} \mathrm{e}^{-} \mathrm{CM}$ frame, if particles are outside the detector acceptance.

In the computation of the $\mathscr{O}(\alpha)$ corrections to the total hadronic cross section, we include the virtual electroweak corrections to $q \bar{q}$ final states, and the real radiation corrections from $q \bar{q} \gamma$ final states, provided the above event-selection criteria are fulfilled. The corrections to the eventshape distributions receive contributions from the virtual electroweak corrections to the $q \bar{q} \mathrm{~g}$ final state, the virtual QCD corrections to the $q \bar{q} \gamma$ final state, and from the real radiation $q \bar{q} g \gamma$ final state. To separate the divergent real radiation contributions, we used both the dipole subtraction method [13, 14] and phase-space slicing [15], resulting in two independent implementations. Soft singularities are regularized dimensionally or with infinitesimal photon and gluon masses; they cancel in the sum of virtual and real corrections. Collinear singularities from initial-state radiation (ISR) are only partially cancelled. The left-over collinear ISR singularity is regularized by the electron mass and absorbed into the initial-state radiator function, which we consider either at fixed order, or in a leading-logarithmic (LL) resummation [16]. Owing to the specific nature of the event selection, collinear divergences from final-state radiation (FSR) are only partially cancelled. The left-over FSR singularity arises from the isolated photon definition, which vetoes on photon jets with $z_{\gamma}>z_{\gamma, \text { cut }}$. This singularity is absorbed into the photon fragmentation function, which we apply in the fixed-order approach of Ref. [17]. For the non-perturbative contribution to this function, we use the $\mathscr{O}(\alpha)$ two-parameter fit of ALEPH [18]. The fragmentation contribution derived in Ref. [17] is based on phase-space slicing and dimensional regularization. We recomputed this contribution using subtraction and mass regularization [14]. 
The one-loop diagrams are generated with FEYNARTS [19]. Using two independent inhouse MATHEMATICA routines, one of which builds upon FORMCALC [20], each diagram is expressed in terms of standard matrix elements and coefficients of tensor integrals. The tensor coefficients are numerically reduced to standard scalar integrals using the methods of Ref. [22]. The scalar master integrals are evaluated using the methods and results of Ref. [23], where UV divergences are regularized dimensionally. For IR divergences two alternative regularizations are employed, one that is fully based on dimensional regularization with massless light fermions, gluons, and photons, and another that is based on infinitesimal photon and gluon masses and small fermion masses. The loop integrals are translated from one scheme to the other as described in Ref. [24].

The Z-boson resonance is described in the complex-mass scheme [25], and its mass is fixed from the complex pole. The electromagnetic couplings appearing in $\mathrm{LO}$ are parametrized in the $G_{\mu}$ scheme, i.e., they are fixed via $\alpha=\alpha_{G_{\mu}}=\sqrt{2} G_{\mu} M_{\mathrm{W}}^{2}\left(1-M_{\mathrm{W}}^{2} / M_{\mathrm{Z}}^{2}\right) / \pi$. As the leading electromagnetic corrections are related to the emission of real photons, we fix the electromagnetic coupling appearing in the relative corrections by $\alpha=\alpha(0)$, which is the appropriate choice for the leading photonic corrections. Accordingly the cross section for $\mathrm{e}^{+} \mathrm{e}^{-} \rightarrow q \bar{q} \mathrm{~g}$ is proportional to $\alpha_{G_{\mu}}^{2} \alpha_{\mathrm{s}}$ while the electroweak corrections to this process are proportional to $\alpha(0) \alpha_{G_{\mu}}^{2} \alpha_{\mathrm{s}}$. The precise numerical input of our evaluations can be found in Ref. [11].

We performed two independent calculations of all ingredients resulting in two independent FORTRAN codes, one of them being an extension of POLE [21].

\section{Results on the normalized thrust distribution}

In Fig. 1, we display the differential thrust distribution normalised to the total hadronic cross section at different $\mathrm{CM}$ energies including NLO electroweak corrections and the relative corrections separately. The distributions are weighted by $(1-T)$, evaluated at each bin centre. The corrections are obtained according to the expansion (2.1), retaining only terms up to LO in $\alpha_{s}$. The Born contribution is given by the $\bar{A}$-term of (2.1), while the full $\mathscr{O}(\alpha)$ corrections contain the treelevel $q \bar{q} \gamma$ contribution $\delta_{\gamma}$ and the NLO electroweak contribution $\delta_{\mathrm{EW}}(2.3)$. With "weak $\mathscr{O}(\alpha)$ " we denote the electroweak NLO corrections without purely photonic corrections, and "h.o. LL" indicates the inclusion of the higher-order ISR effects.

As discussed in Ref. [11] for the Z pole in more detail, large ISR corrections cancel upon normalizing the event shape distribution to the hadronic cross section, resulting in electroweak corrections of a few per cent. Moreover, effects from ISR resummation are largely reduced as well. Note that the photonic corrections develop a distinctive peak structure of up to $9 \%$ in size inside the thrust distribution, an effect that is a remnant of the radiative return to the $\mathrm{Z}$ pole, which is suppressed, but not fully excluded, by the event selection cuts. The purely weak corrections are below 0.5 per mille at the $\mathrm{Z}$ pole, and only grow to the per-cent level for the linear-collider energy of $\sqrt{s}=500 \mathrm{GeV}$.

Data on event-shape distributions and jet cross sections have been corrected for photonic radiation effects modelled by standard LL parton-shower Monte Carlo programs. They can thus not be compared directly with the NLO electroweak corrections computed here. Incorporation of these corrections requires a more profound reanalysis of LEP data, in order to quantify the impact of 

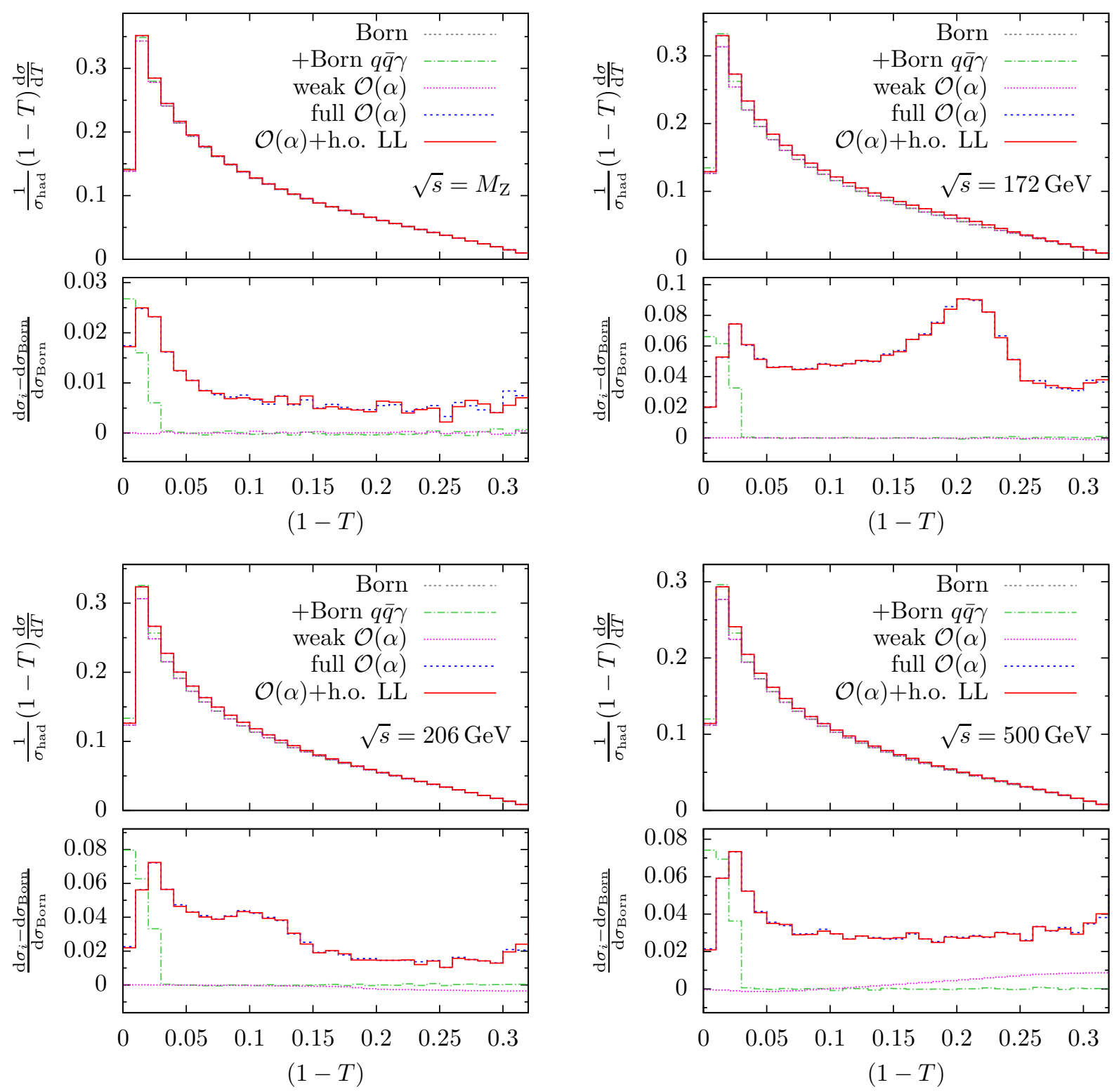

Figure 1: Differential thrust distribution normalised to $\sigma_{\text {had }}$ at different CM energies $\sqrt{s}$.

the NLO electroweak corrections on precision QCD studies, such as the precise extraction of the strong coupling constant at NNLO in QCD [7].

\section{References}

[1] R.K. Ellis, W.J. Stirling and B.R. Webber, QCD and Collider Physics, Cambridge University Press (Cambridge, 1996); G. Dissertori, I.G. Knowles and M. Schmelling, Quantum Chromodynamics: High Energy Experiments and Theory, Oxford University Press (Oxford, 2003).

[2] A. Gehrmann-De Ridder, T. Gehrmann, E.W.N. Glover and G. Heinrich, Phys. Rev. Lett. 99 (2007) 132002 [0707.1285]; JHEP 0712 (2007) 094 [0711.4711]; JHEP 0905 (2009) 106 [0903.4658].

[3] S. Weinzierl, JHEP 0906 (2009) 041 [0904.1077]. 
[4] A. Gehrmann-De Ridder, T. Gehrmann, E.W.N. Glover and G. Heinrich, JHEP 0711 (2007) 058 [0710.0346]; Phys. Rev. Lett. 100 (2008) 172001 [0802.0813].

[5] S. Weinzierl, Phys. Rev. Lett. 101 (2008) 162001 [0807.3241]; JHEP 0907 (2009) 009 [0904.1145].

[6] T. Gehrmann, G. Luisoni and H. Stenzel, Phys. Lett. B 664 (2008) 265 [arXiv:0803.0695].

[7] G. Dissertori, et al., JHEP 0802 (2008) 040 [arXiv:0712.0327]; JHEP 0908 (2009) 036 [arXiv:0906.3436]; R.A. Davison and B.R. Webber, Eur. Phys. J. C 59 (2009) 13 [arXiv:0809.3326]; S. Bethke, S. Kluth, C. Pahl and J. Schieck [JADE Collaboration], Eur. Phys. J. C 64 (2009) 351 [arXiv:0810.1389]; T. Gehrmann, M. Jaquier and G. Luisoni, arXiv:0911.2422.

[8] G. Dissertori, et al., arXiv:0910.4283.

[9] T. Becher and M. D. Schwartz, JHEP 0807 (2008) 034 [arXiv:0803.0342 [hep-ph]].

[10] C.M. Carloni-Calame, S. Moretti, F. Piccinini and D.A. Ross, JHEP 0903 (2009) 047 [0804.3771], Eur. Phys. J. C 62 (2009) 355 [0811.4758], 0903.0490.

[11] A. Denner, S. Dittmaier, T. Gehrmann and C. Kurz, Phys. Lett. B 679 (2009) 219 [arXiv:0906.0372 [hep-ph]].

[12] A. Heister et al. [ALEPH Collaboration], Eur. Phys. J. C 35 (2004) 457.

[13] S. Catani and M.H. Seymour, Nucl. Phys. B 485 (1997) 291 [hep-ph/9605323]; 510 (1997) 503(E).

[14] S. Dittmaier, Nucl. Phys. B 565 (2000) 69 [hep-ph/9904440]; S. Dittmaier, A. Kabelschacht and T. Kasprzik, Nucl. Phys. B 800 (2008) 146 [0802.1405].

[15] W.T. Giele and E.W.N. Glover, Phys. Rev. D 46 (1992) 1980; W.T. Giele, E.W.N. Glover and D.A. Kosower, Nucl. Phys. B 403 (1993) 633 [hep-ph/9302225].

[16] W. Beenakker et al., in Physics at LEP2, eds. G. Altarelli, T. Sjöstrand and F. Zwirner (CERN 96-01, Geneva, 1996), Vol. 1, p. 79, hep-ph/9602351.

[17] E.W.N. Glover and A.G. Morgan, Z. Phys. C 62 (1994) 311.

[18] D. Buskulic et al. [ALEPH Collaboration], Z. Phys. C 69 (1996) 365.

[19] J. Küblbeck, M. Böhm and A. Denner, Comput. Phys. Commun. 60 (1990) 165; H. Eck and J. Küblbeck, Guide to FeynArts 1.0, University of Würzburg, 1992; T. Hahn, Comput. Phys. Commun. 140 (2001) 418 [hep-ph/0012260].

[20] T. Hahn and M. Pérez-Victoria, Comput. Phys. Commun. 118 (1999) 153 [hep-ph/9807565]; T. Hahn, Nucl. Phys. Proc. Suppl. 89 (2000) 231 [hep-ph/0005029].

[21] E. Accomando, A. Denner and C. Meier, Eur. Phys. J. C 47 (2006) 125 [arXiv:hep-ph/0509234].

[22] A. Denner and S. Dittmaier, Nucl. Phys. B 658 (2003) 175 [hep-ph/0212259] and Nucl. Phys. B 734 (2006) 62 [hep-ph/0509141].

[23] G. 't Hooft and M. Veltman, Nucl. Phys. B 153 (1979) 365; W. Beenakker and A. Denner, Nucl. Phys. B 338 (1990) 349; A. Denner, U. Nierste and R. Scharf, Nucl. Phys. B 367 (1991) 637.

[24] S. Dittmaier, Nucl. Phys. B 675 (2003) 447 [hep-ph/0308246].

[25] A. Denner, S. Dittmaier, M. Roth and D. Wackeroth, Nucl. Phys. B 560 (1999) 33 [hep-ph/9904472]; A. Denner, S. Dittmaier, M. Roth and L. H. Wieders, Nucl. Phys. B 724 (2005) 247 [hep-ph/0505042].

[26] D. Y. Bardin et al., in Z Physics at LEP1, eds. G. Altarelli, R. Kleiss and C. Verzegnassi (CERN 89-08, Geneva, 1989), Vol. 1, p. 89, 